\title{
Effects of hemodynamic monitoring using a single-use transesophageal echocardiography probe in critically ill patients - study protocol for a randomized controlled trial
}

\author{
Luca Cioccari ${ }^{1}$, Bjoern Zante ${ }^{1}$, Andreas Bloch¹, David Berger ${ }^{1}$, Andreas Limacher ${ }^{2}$, Stephan M. Jakob',
} Jukka Takala ${ }^{\top}$ and Tobias M. Merz ${ }^{*}$

\begin{abstract}
Background: Hemodynamic instability is one of the leading causes of intensive care unit (ICU) admission. Early stabilization of hemodynamics is associated with improved outcome. The monitoring used to guide hemodynamic support may influence the time needed to achieve stable hemodynamics. Visualization of the heart using echocardiography offers the advantage of direct measurement of cardiac volumes and ventricular function. A miniaturized monoplane transesophageal echocardiography (TEE) probe was developed, allowing for almost continuous qualitative hemodynamic TEE assessment (hTEE) after brief bedside training. The primary objective of the study is to assess whether hemodynamic monitoring using the hTEE technology shortens time to resolution of shock in ICU patients in comparison to standard monitoring using a central venous catheter, pulmonary artery catheter, or conventional echocardiography.

Methods: Five hundred consecutive subjects with circulatory shock (low mean arterial blood pressure (MAP) and signs of organ hypoperfusion) at the time of ICU admission are included in the study. The subjects are randomly assigned to one of four groups using a $2 \times 2$ factorial design stratified by method of hemodynamic monitoring ( $\mathrm{hTEE}$ vs standard hemodynamic monitoring) and frequency of hemodynamic assessments (minimum every $4 \mathrm{~h}$ vs standard of care). The primary study outcome is the time from study inclusion to resolution of circulatory shock, defined as MAP > $60 \mathrm{mmHg}$ for $\geq 4 \mathrm{~h}$ after discontinuation of vasopressors and inotropes. The hTEE monitoring consists of the acquisition of three defined echocardiography views: Transgastric mid-esophageal short axis with measurement of fractional area change of left ventricle, mid-esophageal four-chamber view with measurement of the ratio of right to left ventricular area, and mid-esophageal ascending aortic short-axis view with measurement of the superior vena cava collapsibility index. In the control groups, monitoring modalities, including conventional TTE and TEE but not hTEE, are at the discretion of the treating physician. The interpretation of hemodynamic monitoring and the subsequent changes in patient management are recorded after each hemodynamic assessment. Differences in the primary and further secondary time-to-event outcomes will be assessed using a competing risk model accounting for the competing risk of death.
\end{abstract}

(Continued on next page)

\footnotetext{
*Correspondence: tobias.merz@insel.ch

${ }^{1}$ Department of Intensive Care Medicine, University Hospital, and University

of Bern, 3010 Bern, Switzerland

Full list of author information is available at the end of the article
}

(c) The Author(s). 2018 Open Access This article is distributed under the terms of the Creative Commons Attribution 4.0 International License (http://creativecommons.org/licenses/by/4.0/), which permits unrestricted use, distribution, and reproduction in any medium, provided you give appropriate credit to the original author(s) and the source, provide a link to the Creative Commons license, and indicate if changes were made. The Creative Commons Public Domain Dedication waiver (http://creativecommons.org/publicdomain/zero/1.0/) applies to the data made available in this article, unless otherwise stated. 
(Continued from previous page)

Discussion: The effect of using echocardiography as a monitoring modality on relevant patient outcomes has not been established so far. The study at hand may be one of the first trials to provide detailed data on effectiveness and safety of echocardiography to guide treatment in patients with circulatory shock.

Trial registration: ClinicalTrials.gov, ID: NCT02048566. Registered on January 29, 2014.

Keywords: Circulatory shock, Intensive care unit, Hemodynamic monitoring, Echocardiography,

\section{Background}

Hemodynamic management of critically ill patients is a constant challenge in the intensive care unit (ICU). Available monitoring parameters to guide hemodynamic management consist of measurements of pressures (systemic and pulmonary artery pressures, central venous pressure) and flow (cardiac output measurements). However, cardiac filling pressure data have known limitations and might not accurately represent cardiac preload and contractility [1]. To date, continuous or sequential recording of cardiac output parameters is limited to pulse contour analysis and indicator-dilution techniques [2]. These methods allow only an incomplete characterization of the patient's cardio-circulatory status and comparisons of different measurements of cardiac function parameters are reported to trend differently in response to therapy and to show limited inter-device agreement [3]. Hemodynamic management of critically ill patients based on these parameters might, therefore, not be optimal and delay the stabilization of the patient, leading to worse outcomes and increased use of resources [4].

Echocardiography is an established tool to evaluate the causes of hemodynamic instability in ICU patients by the visualization of cardiac chambers, valves and pericardium and of cardiac functional abnormalities $[5,6]$. Transthoracic (TTE) or transesophageal (TEE) echocardiography can be used as a first-line approach for a quick and focused examination [7]. However, the training necessary to reliably perform focused echocardiography is substantial $[8,9]$ and the method is not readily available for every intensivist. A repeated echocardiographic assessment could potentially provide useful additional information resulting in more rapid resolution of hemodynamic instability [10]. Monitoring hemodynamics with conventional TTE and TEE is not feasible due to a lack of time and availability of appropriately trained staff.

A miniaturized monoplane TEE probe that can be left inserted in the esophagus for up to 3 days has been recently developed. Qualitative hemodynamic TEE assessment (hTEE) using the miniaturized probe allows for almost continuous echocardiographic monitoring of unstable ICU patients [11], and can provide useful additional information for hemodynamic management when compared to standard, non-ultrasound-based monitoring modalities [10]. The feasibility of such qualitative hTEE by intensivists after a 6 - $\mathrm{h}$ bedside training has been demonstrated [12]. However, as of yet, studies assessing the impact of hemodynamic monitoring by hTEE on relevant patient outcomes are not available. Given the associated costs for the hTEE device and the ultrasound probes and the additional resource requirements for training and use, the efficacy and efficiency of hTEE monitoring in comparison to standard monitoring should be established.

\section{Methods \\ Hypothesis}

The primary study hypothesis is that hemodynamic monitoring using hTEE in critically ill patients receiving cardiovascular support allows for a shorter time to resolution of circulatory shock compared to standard ICU monitoring alone, using a central venous catheter, pulmonary artery catheter, or conventional TTE and TEE. In this context, we define time to resolution of shock as the time from randomization to the time point of resolution of circulatory shock (first time point fulfilling the definition mean arterial pressure (MAP) $\geq 60 \mathrm{mmHg}$ for at least $4 \mathrm{~h}$ after discontinuation of vasopressors and inotropes). The secondary hypothesis is that hemodynamic assessment at least every $4 \mathrm{~h}$ results in shorter time to resolution of circulatory shock as compared to standard hemodynamic assessment (at least once per nursing shift).

\section{Objectives}

The primary objective of the study is to assess whether hemodynamic monitoring using the hTEE technology shortens time to resolution of shock in ICU patients in comparison to standard monitoring using a central venous catheter, pulmonary artery catheter, or conventional echocardiography. Secondary objectives include whether hemodynamic assessment at mandated 4-hourly intervals shortens the time to resolution of shock in comparison to standard assessment intervals, as well as the safety and tolerability of the hTEE probe.

\section{Outcomes}

The primary study outcome is the time from study inclusion to resolution of circulatory shock (as defined above). The secondary outcomes include the time to resolution of 
clinical signs of shock (capillary refilling time $<3 \mathrm{~s}$, urine output $>0.5 \mathrm{~mL} / \mathrm{kg} / \mathrm{h}$ for at least $4 \mathrm{~h}$, blood lactate < $2 \mathrm{mmol} / \mathrm{L}$ )), the length of time on organ support (mechanical ventilation, renal-replacement therapy), length of stay (LOS) in the ICU and in hospital, ICU and hospital mortality, and the use of conventional hemodynamic monitoring (pulmonary artery catheter, central venous catheter, conventional echocardiography) and occurrence of SAE. A detailed description of study outcomes and adverse events is included in Additional file 1.

\section{Trial design}

This is a randomized, open label, $2 \times 2$ factorial design, controlled clinical trial comparing the effect of hemodynamic monitoring using the hTEE technology (ImaCor ClariTEE system IMACOR, New York, NY, USA) or standard hemodynamic monitoring in a single center. Patients with circulatory shock, defined as persistent hypotension despite adequate fluid resuscitation and signs of hypoperfusion or organ dysfunction (at least one of the following: capillary refilling time $3 \mathrm{~s}$ or longer, urine output $<0.5 \mathrm{~mL} / \mathrm{kg}$ for at least $1 \mathrm{~h}$, lactate $>2 \mathrm{mmol} / \mathrm{L}$ ) are included into the study at the time of ICU emergency admission. Subjects are randomized into four groups using a $2 \times 2$ factorial study design. The four groups are stratified by method of hemodynamic monitoring (hTEE vs standard hemodynamic monitoring) and frequency of hemodynamic assessments (protocolized maximal interval of $4 \mathrm{~h}$ vs standard monitoring intervals with maximal interval of $8 \mathrm{~h}$ ). Assessed factors are use of hTEE monitoring vs conventional monitoring and use of protocolized intervals for hemodynamic assessment vs standard assessment intervals. The Standard Protocol Items Recommendations for Interventional Trials (SPIRIT) checklist is attached as Additional file 2.

\section{Study setting}

The study is performed at the Department of Intensive Care Medicine of the University Hospital Bern, a 60-bed ICU in a 960-bed tertiary care center in Switzerland.

\section{hTEE operators}

ICU specialists perform the study examinations. All hTEE operators receive a total of $4 \mathrm{~h}$ training in the use of hTEE by an experienced operator. Training includes an introduction to the method and a demonstration of the device use in the context of a presentation, followed by one-to-one bedside training of all necessary skills to use the hTEE device for acquiring and interpreting images in the study context.

\section{Patient eligibility criteria}

All ICU patients are screened at the time of ICU admission for eligibility for the study. All ICU admissions of the last $24 \mathrm{~h}$ are additionally and independently screened to identify missed study patients each day at 8 a.m. to achieve adequate patient enrollment. Subjects of 18 years of age or older who require mechanical ventilation through an endotracheal, naso-tracheal, or tracheostomy tube with circulatory shock of any cause are included in the study. Circulatory shock is defined as follows:

- Systemic mean arterial blood pressure $<60 \mathrm{mmHg}$ (or $<80 \mathrm{mmHg}$ if the patient has baseline hypertension) for more than $30 \mathrm{~min}$ despite adequate fluid resuscitation (minimum of $20 \mathrm{~mL} / \mathrm{kg}$ of crystalloids) or maintaining the systemic mean arterial blood pressure $\geq 60 \mathrm{mmHg}$ requires any dose of vasopressors (norepinephrine, vasopressin) or inotropes (epinephrine, dobutamine, milrinone, aminophylline)

- Signs of hypoperfusion or organ dysfunction (at least one of the following: capillary refilling time $3 \mathrm{~s}$ or longer, urine output $<0.5 \mathrm{~mL} / \mathrm{kg}$ for at least $1 \mathrm{~h}$, lactate $>2 \mathrm{mmol} / \mathrm{L}$ )

\section{Patient exclusion criteria}

Exclusion criteria consist of pathologies of the upper gastrointestinal tract (unrepaired trachea-esophageal fistula, history of prior esophageal or gastric surgery precluding the use of TEE, esophageal obstruction, stricture, varices or diverticulum, esophageal or gastric perforation or esophageal bleeding, vascular ring, aortic arch anomaly with or without airway compromise, recent oropharyngeal surgery) and severe coagulopathy (thrombocyte count less than $30 \times 10^{9} / \mathrm{L}$ or INR $>3$ ). Additionally, patients with cervical spine injury or anomaly, patients after elective ICU admission after planned surgery and patients with cardiac assist devices (intra-aortal balloon pump, ventricular assist devices, extra-corporeal membrane oxygenation) are not included in the study.

\section{Allocation}

The allocation sequence was generated before commencement of the study by ICU research staff using computer-generated random numbers with randomly varying block sizes of 4,8 and 12 [13]. Allocations concealment is ensured using sequentially numbered, identical, opaque, sealed envelopes. Patients are randomized and enrolled by the admitting on-call ICU specialist after reviewing the inclusion and exclusion criteria and after obtaining confirmation by a physician who is not participating in study that the interests of the patient are safeguarded. Due to logistical reasons (two hTEE devices available for the study) inclusion of a maximum of two patients into the groups applying hTEE is possible at the same time. Randomization and recruitment is interrupted as soon as a second patient is randomized into a treatment 
group in which hTEE is applied. Randomization and recruitment is restarted as soon as at least one hTEE device is available for further patients. Blinding of health care providers is not feasible which means that all clinical staff caring for the patients are aware of the allocation during the study period.

\section{Interventions and study procedures}

The subjects are assigned to one of four groups stratified by method of hemodynamic monitoring (hTEE vs control hemodynamic monitoring) and frequency of hemodynamic assessments (protocolized maximal interval of $4 \mathrm{~h}$ vs standard monitoring intervals with maximal interval of $8 \mathrm{~h}$ ) (Table 1):

In patients randomized to echocardiography-guided hemodynamic management (hTEEPM and hTEESM) the ImaCor ClariTEE system (IMACOR, New York, NY, USA) is installed at the time of study inclusion by the ICU specialist in charge of the patient. The device produces a single-plane two-dimensional image and has color Doppler capability. The hTEE probe is a $5.5-\mathrm{mm}$ detachable probe; due to its small size, it can remain in situ for up to $72 \mathrm{~h}$ and, therefore, allows for reassessment of the patient's hemodynamic progress and the effect of selected interventions at any time. Before insertion, the probe is connected to a dedicated echocardiographic system, which allows the recording of digital loops and the performance of basic two-dimensional measurements of areas and distances. Additional hemodynamic monitoring modalities are restricted to techniques routinely used at the study center (central venous catheter, pulmonary artery catheter, and conventional echocardiography) and can be used at the discretion of the ICU specialist in charge. After positioning of the hTEE probe, the following three hTEE standard views and measurements are acquired and performed:
- transgastric mid-esophageal short-axis view (TG mid SAX); fractional area change (FAC) of left ventricle (LV)

- Mid-esophageal four-chamber view (ME fourchamber); ratio of right ventricular (RV) to left ventricular area (RVEDA/LVEDA ratio)

- Mid-esophageal ascending aortic short-axis view (ME asc aortic SAX); collapsibility index of superior vena cava (SVC CI)

Left ventricular areas at end-systole (LVESA) and at end-diastole (LVEDA) are measured from the TG mid SAX view, the fractional area change (FAC) of the left ventricle (LV) is calculated as LVEDA/LVESA and used to grade left ventricular ejection fraction as normal (FAC > $50 \%$ ), moderately decreased (FAC $40-50 \%$ ) or severely decreased (FAC $<40 \%)$. Similarly, calculation of the RV/LV ratio is performed by measuring left and right ventricular areas at end-diastole in the ME four-chamber view. A ratio $>0.6$ is used as indicator of right ventricular dysfunction. The collapsibility of the superior vena cava (SVC) is rated by calculating the collapsibility index, i.e., the inspiratory decrease in SVC diameter. The index is determined as (maximal diameter on expiration - minimal diameter on inspiration)/maximal diameter on expiration, expressed as a percentage [14]. A threshold of $>35 \%$ is used to distinguish between the presence and absence of hypovolemia. After image acquisition, the ICU specialist answers the following questions about quantification/interpretation of all hemodynamic monitoring and subsequent changes in hemodynamic management using a study case report form (CRF) (Table 2):

In patients randomized to conventional hemodynamic management (ControlPM and ControlSM) hemodynamic monitoring is established at the discretion of the ICU specialist in charge. The use of hTEE is excluded in this group, whereas the use of conventional TTE or TEE is

Table 1 Study groups

\begin{tabular}{|c|c|c|c|}
\hline Study group & Hemodynamic monitoring & Monitoring interval & $n$ \\
\hline $\begin{array}{l}\text { hTEE protocolized monitoring } \\
\text { (hTEEPM) }\end{array}$ & $\begin{array}{l}\text { - hTEE-guided hemodynamic management } \\
\text { - Additional hemodynamic monitoring of } \\
\text { choice of the treating physician }\end{array}$ & $\begin{array}{l}\text { - Study inclusion } \\
\text { - Time of occurrence of new organ system deterioration } \\
\text { - At least every } 4 \mathrm{~h}\end{array}$ & 125 \\
\hline $\begin{array}{l}\text { hTEE standard monitoring } \\
\text { (hTEESM) }\end{array}$ & $\begin{array}{l}\text { - hTEE-guided hemodynamic management } \\
\text { - Additional hemodynamic monitoring of } \\
\text { choice of the treating physician }\end{array}$ & $\begin{array}{l}\text { - Study inclusion } \\
\text { - Follow-up measurement time points at the discretion } \\
\text { of the treating physician } \\
\text { - At least every } 8 \mathrm{~h} \text { (standard of care in our ICU) }\end{array}$ & 125 \\
\hline $\begin{array}{l}\text { Control protocolized monitoring } \\
\text { (ControlPM) }\end{array}$ & $\begin{array}{l}\text { - Any hemodynamic monitoring of choice } \\
\text { of the treating physician except hTEE }\end{array}$ & $\begin{array}{l}\text { - Study inclusion } \\
\text { - Time of occurrence of new organ system deterioration } \\
\text { - At least every } 4 \mathrm{~h}\end{array}$ & 125 \\
\hline $\begin{array}{l}\text { Control standard monitoring } \\
\text { (ControlSM) }\end{array}$ & $\begin{array}{l}\text { - Any hemodynamic monitoring of choice } \\
\text { of the treating physician except hTEE }\end{array}$ & $\begin{array}{l}\text { - Study inclusion } \\
\text { - Follow-up measurement time points at the discretion } \\
\text { of the treating physician } \\
\text { - At least every } 8 \mathrm{~h} \text { (standard of care in our intensive } \\
\text { care unit (ICU)) }\end{array}$ & 125 \\
\hline
\end{tabular}


Table 2 Quantification/interpretation of hemodynamic monitoring and changes in hemodynamic management

\begin{tabular}{|c|c|}
\hline Quantification/interpretation of all hemodynamic monitoring & $\begin{array}{l}\text { Changes in hemodynamic management based on information } \\
\text { acquired by hemodynamic monitoring? }\end{array}$ \\
\hline $\begin{array}{l}\text { - Systolic LV function: normal - moderately decreased - severely decreased? } \\
\text { - RV dysfunction: present -absent? } \\
\text { - Hypovolemia: present - absent? } \\
\text { - Clinically significant pericardial effusion: present - absent? } \\
\text { guide hemodyation acquired by hemodynamic monitoring useful to } \\
\text { - Is there need for further monitoring? Yes - no? }\end{array}$ & $\begin{array}{l}\text { - No changes } \\
\text { - Additional fluids given } \\
\text { - Start/increase of inotropes (epinephrine, dobutamine, milrinone, } \\
\text { aminophylline) } \\
\text { - Stop/decrease inotropes (epinephrine, dobutamine, milrinone, } \\
\text { aminophylline) } \\
\text { - Start/increase of vasopressors (norepinephrine, vasopressin) } \\
\text { - Stop/decrease of vasopressors (norepinephrine, vasopressin) } \\
\text { - Drainage of pericardial effusion } \\
\text { - Other (to be specified) }\end{array}$ \\
\hline
\end{tabular}

$R V$ right ventricular, $L V$ left ventricular

permitted. After hemodynamic assessment, the ICU specialist answers the same questions about quantification/interpretation of all hemodynamic monitoring and subsequent changes in hemodynamic management as in groups hTEEPM and hTEESM (Table 2).

Study procedures and measurements in all groups are performed until the primary outcome (resolution of shock) occurs or for a maximum of $72 \mathrm{~h}$. Criteria for discontinuing hTEE monitoring are any occurrence of complications potentially attributed to the use of hTEE such as oral, pharyngeal, esophageal, or gastrointestinal bleeding or injury and cardiac arrhythmias (supraventricular/ventricular tachycardia, atrioventricular block, asystole) attributed to the presence of an hTEE probe by the treating physician. For the control groups (ControlPM, ControlSM) criteria for discontinuing the allocated intervention have not been defined as they receive standard intensive care. Adherence to the study protocol is monitored for each included patient by daily monitoring of completion of study CRF by the research staff. Concomitant care for all study groups consists of standard interventions in the context of the routine care of ICU patients as per the decision of the treating ICU specialist. The ICU uses a cardiovascular management protocol to guide hemodynamic stabilization [15]. Commonly used monitoring modalities consist of measurement of arterial and central venous pressure, use of pulmonary artery catheters with continuous monitoring of cardiac output and mixed venous saturation, and conventional echocardiography. The standard vasopressor is norepinephrine; standard inotropes are dobutamine, milrinone and epinephrine. Pulse-contour analysis modalities are not applied.

\section{Participant timeline}

The time schedule of enrollment, interventions and assessments is outlined in Fig. 1. Recruitment of study subjects started in March 2014 and is ongoing.

\section{Sample size}

Sample size calculation is based on a retrospective analysis of a sample of 159 patients admitted to our ICU during a 3-month period, which fulfilled the study entry criteria. Median time to resolution of circulatory shock, defined as mean systemic blood pressure $>60 \mathrm{mmHg}$ and resolution of clinical signs of shock (capillary refilling time $<3 \mathrm{~s}$, urine output $>0.5 \mathrm{~mL} / \mathrm{kg} / \mathrm{h}$ for at least $4 \mathrm{~h}$, blood lactate $<2 \mathrm{mmol} / \mathrm{L}$ ), in this sample was $18.5 \mathrm{~h}$ (Fig. 1). We used the Stata command artsurv to calculate the sample size [16]. We applied the unweighted log-rank test and derived expected probabilities of hemodynamic stabilization and loss-to-follow up (i.e., death) over time from the retrospective analysis. To identify a clinically relevant reduction of time to resolution of circulatory shock of $25 \%$ (i.e., from 18.5 to $14.0 \mathrm{~h}$ ) we calculated a required sample size of 458 patients to achieve a power of $80 \%$ at a two-sided alpha level of 0.05 for the main effect (comparison of monitoring with/without hTEE). In order to allow for study drop out, the inability to consent, or the withdrawal of consent, we choose a sample size of 125 patients for each of the four groups or 500 patients in total. Patient recruitment will be continued until a total of 500 patients with a complete study follow-up, and in which consent for study inclusion has been obtained by the patient, are included into the study.

\section{Data collection methods}

In all study patients, hemodynamic parameters, use of vasopressors/inotropes as continuous intravenous infusions, parameters determined by organ support (mechanical ventilation, renal-replacement therapy) and results of blood sampling are registered automatically by the electronic patient data management system (PDMS) as part of the routine patient data collection. Capillary refill time (hourly) and ICU resource use (accumulated TISS points, hourly) and urinary output (2-hourly) are part of the routine data collection performed by the bedside ICU nurse; these data are manually entered into the PDMS. LOS in hospital and hospital mortality are extracted from the hospital database. Blood lactate levels are measured in study patients at least every $2 \mathrm{~h}$. A paper-based study CRF is the primary data collection instrument for the study. All 


\begin{tabular}{|c|c|c|c|c|c|c|c|c|}
\hline \multirow[b]{3}{*}{ Time point } & \multicolumn{8}{|c|}{ Study period } \\
\hline & \multirow{2}{*}{$\begin{array}{c}\text { Enrolment } \\
-t_{1}\end{array}$} & \multirow{2}{*}{$\begin{array}{c}\begin{array}{c}\text { Allocation } \\
\text { and study } \\
\text { inclusion }\end{array} \\
T_{0}\end{array}$} & \multicolumn{5}{|c|}{ Post-allocation } & \multirow{2}{*}{$\begin{array}{c}\begin{array}{c}\text { Close- } \\
\text { out }\end{array} \\
T_{0+72 h}\end{array}$} \\
\hline & & & $T_{0+4 h}$ & $T_{0+8 h}$ & $T_{0+12 h}$ & $\mathrm{~T}_{0+16 \mathrm{hh}}$ & etc. & \\
\hline \multicolumn{9}{|l|}{ Enrolment } \\
\hline Eligibility screen & $\mathrm{x}$ & & & & & & & \\
\hline $\begin{array}{l}\text { Confirmation by } \\
\text { independent } \\
\text { physician }\end{array}$ & $x$ & & & & & & & \\
\hline $\begin{array}{l}\text { Proxy deferred } \\
\text { consent }\end{array}$ & & & \multicolumn{5}{|c|}{ Within $72 \mathrm{~h}$ after enrolment } & \\
\hline $\begin{array}{l}\text { Patient deferred } \\
\text { consent }\end{array}$ & & & \multicolumn{5}{|c|}{$\begin{array}{c}\text { As soon as patient's condition } \\
\text { allows }\end{array}$} & \\
\hline Allocation & & $\mathrm{X}$ & & & & & & \\
\hline \multicolumn{9}{|l|}{ Assessments: } \\
\hline $\begin{array}{l}\text { Group hTEEPM } \\
\text { hTEE assessment }\end{array}$ & & $x$ & $\begin{array}{c}\mathrm{X} \\
\text { Additi } \\
\text { syster }\end{array}$ & $\begin{array}{c}\mathrm{X} \\
\text { onal as } \\
\text { n dete } \\
\end{array}$ & $\begin{array}{c}\mathrm{X} \\
\text { sessme } \\
\text { ioration }\end{array}$ & $\begin{array}{l}\quad x \\
\text { t if new } \\
\text { occurs }\end{array}$ & $\begin{array}{l}\text { etc. } \\
\text { rgan }\end{array}$ & $x$ \\
\hline $\begin{array}{l}\text { Group hTEESM } \\
\text { hTEE assessment }\end{array}$ & & $\mathrm{x}$ & $\begin{array}{l}\text { Additi } \\
\text { discre }\end{array}$ & $\begin{array}{c}\mathrm{X} \\
\text { onal as } \\
\text { tion of }\end{array}$ & $\begin{array}{l}\text { sessme } \\
\text { the trea }\end{array}$ & $\begin{array}{l}\quad X \\
\text { ts at the } \\
\text { ting phys }\end{array}$ & cian & $x$ \\
\hline $\begin{array}{l}\text { Group ControlPM } \\
\text { standard } \\
\text { assessment }\end{array}$ & & $x$ & $\begin{array}{c}\mathrm{X} \\
\text { Additi } \\
\text { syster }\end{array}$ & $\begin{array}{c}\mathrm{X} \\
\text { onal as } \\
\text { n dete }\end{array}$ & $\begin{array}{l}\qquad X \\
\text { sessme } \\
\text { ioration }\end{array}$ & \begin{tabular}{l}
\multicolumn{1}{c}{$\mathrm{X}$} \\
it if new \\
occurs
\end{tabular} & $\begin{array}{l}\text { etc. } \\
\text { rgan }\end{array}$ & $x$ \\
\hline $\begin{array}{l}\text { Group ControlSM } \\
\text { standard } \\
\text { assessment }\end{array}$ & & $x$ & $\begin{array}{l}\text { Additi } \\
\text { discre }\end{array}$ & $\begin{array}{l}\mathrm{X} \\
\text { onal as } \\
\text { tion of }\end{array}$ & $\begin{array}{l}\text { sessme } \\
\text { the trea }\end{array}$ & $\begin{array}{l}\quad \mathrm{X} \\
\text { its at the } \\
\text { ting phys }\end{array}$ & cian & $x$ \\
\hline
\end{tabular}

Fig. 1 Participant timeline

study data necessary for statistical analysis according to the SAP are recorded in the CRF. All data requested on the CRF are recorded during hemodynamic assessment. If a space on the CRF is left blank because the procedure was not done or the question was not asked, "N/D" is written instead. If an item is not applicable to the individual case, "N/A" is written. All corrections must be initialed and dated. All hTEE echocardiography loops are recorded and saved for independent and blinded off-line review.

\section{Data management}

Data are entered into a web-based electronic CRF. Study subjects are assigned an individual identifying study code on all data documents, which does not contain identifying information. The investigators keep a separate documentation that links the study code to subjects' identifying information locked in a separate location and restrict access to this document to research staff. CRF and electronic data are archived for 10 years.

\section{Statistical methods}

Continuous baseline and procedural variables will be displayed as mean (standard deviation) for normally and median (quartiles) for non-normally distributed data, categorical variables as number (percentage). The primary analysis will be an intention-to-treat (ITT) analysis, i.e., all patients will be analyzed as randomized. We will first test the primary hypothesis, the difference in time to hemodynamic stabilization between the hTEE and standard group. Second, we will evaluate the difference between the protocoled and standard monitoring. Differences in the primary outcome will be assessed using the Fine-Gray competing risk model accounting for the competing risk of death [17]. The model that compares the method of monitoring (hTEE vs standard) will be adjusted for the frequency of monitoring (protocolizedvs standard), the model that compares the frequency of monitoring for the method of monitoring. We will also test for an interaction between the method (hTEE vs 
standard) and frequency of hemodynamic monitoring (protocolized vs standard) in order to assess if effects are different depending on the method and frequency of monitoring. We will enter an interaction term in the model described above. If there is significant interaction, we will present effects separately in subgroups.

The secondary outcome, time to death, will be evaluated using Cox proportional-hazards regression. Other secondary time-to-event outcomes will be analyzed using competing risk models as described above. Binary outcomes will be analyzed using logistic regression, continuous score data using linear regression. We will also perform a secondary per-protocol analysis excluding patients that did not receive the allocated interventions or had major protocol violations. Statistical analyses will be described in more detail in a statistical analysis plan (SAP).

\section{Data monitoring}

The trial is externally monitored (Clinical Trials Unit, Bern, Switzerland) in accordance with Good Clinical Practice (GCP) standards.

\section{Monitoring harm}

Occurrence of adverse events due to prolonged hemodynamic impairment and treatment with vasopressors/inotropes is registered, including ventricular tachycardia, ventricular fibrillation, atrial fibrillation, myocardial infarction as defined in [18], skin necrosis, stroke, secondary bowel or limb ischemia, secondary infections (catheter-related infections, ventilator-associated pneumonia VAP, new onset of sepsis) and gastrointestinal bleeding complications. Additionally, any occurrence of complications potentially attributed to the use of hTEE such as oral, pharyngeal, esophageal, or gastrointestinal bleeding or injury, cardiac arrhythmias occurring while hTEE probe is in place (supraventricular/ventricular tachycardia, atrioventricular block and asystole) and need for additional sedation and/or muscle relaxants for hTEE probe placement and hTEE assessment is recorded. The study includes critically ill patients with circulatory shock. Depending on the reasons for hemodynamic impairment and comorbidities a mortality rate of up to $50 \%$ has to be expected [19]. In accordance with current guidelines, serious adverse events (SAE) and serious adverse device effect (anticipated or unanticipated) will be thoroughly investigated to determine causality and reported to the appropriate authorities (Kantonale Ethikkommission Bern) if a relation between SAE and study procedures is considered unlikely, likely, or certain, or in the event of death of the patient. No SAE report is made if a connection between SAE and study procedures can be excluded. Subjects experiencing any trial-related adverse events will receive the best possible care, including follow-up visits as clinically indicated.

\section{Confidentiality}

Information about study subjects is kept confidential. A signed subject authorization is part of the informed consent documents, informing the subject what protected health information (PHI) is collected from subjects in this study, who will have access to the information and the rights of a research subject to revoke their authorization for use of their PHI. All data are entered into a dedicated study data management system. Study subjects are assigned an individual identifying study code on all data documents (e.g., completed questionnaire) which does not contain identifying information. The investigators keep a separate documentation that links the study code to subjects' identifying information in a separate location.

\section{Discussion}

The targeted patient population fulfills the definition of circulatory shock, representing the most severely ill patients with plausibly the highest possible chance to show a difference between the intervention arms. Echocardiography has proven a useful tool to identify reversible causes of shock and to monitor left and right ventricular function [20]. However, despite several class 1 recommendations for its use in the ICU, the effect of echocardiography as a monitoring modality on relevant patient outcomes has not been studied in large randomized trials so far. The study at hand aims at establishing the effect of frequent hemodynamic TEE monitoring using a miniaturized probe that allows minimizing invasiveness of TEE examinations. The study may be one of the first trials to bridge the gap between evidence and clinical practice and to provide detailed data on efficacy and safety of echocardiography to guide treatment in patients with circulatory shock.

\section{Strengths and limitations}

To our knowledge, this is the largest randomized controlled trial of the effect of hTEE-monitoring in critically ill patients with circulatory shock. The primary outcome is objective and verifiable, and the statistical analysis adjusts for frequency and method of monitoring, including testing for interaction, in order to assess if any difference in outcome depends on the method or on the frequency of monitoring. The feasibility of hTEE monitoring has been previously established and the technology has shown to provide additional valid information for the management of critically ill patients. Our study has some limitations. First, patients and health care providers are not blinded. However, due to the nature of the intervention, blinding is not feasible in this setting. Second, hemodynamic management is not standardized, but remains at the discretion of the treating physician. This is likely to reflect clinical practice and avoids introducing a potential confounder (such as a treatment protocol). Third, the single-center design may limit external validity. However, our unit is a tertiary ICU 
in a large university hospital and, therefore, is likely to reflect current best practice in the management of critically ill patients with shock.

\section{Trial status}

Patient recruitment has started in May 2014, recruitment will likely be completed by December 2017. Protocol V2 01.12.2013.

\section{Additional files}

Additional file 1: Definitions and measurement of outcome parameters and serious adverse events. (DOCX $44 \mathrm{~kb}$ )

Additional file 2: Standard Protocol Items: Recommendations for Interventional Trial (SPIRIT) 2013 Checklist: recommended items to address in a clinical trial protocol and related documents*. (DOC $168 \mathrm{~kb}$ )

\begin{abstract}
Abbreviations
ControlPM: Patient group randomized to control with protocolized monitoring; ControlSM: Patient group randomized to control with standard monitoring; FAC: Fractional area change of left ventricle; hTEE: Qualitative hemodynamic TEE assessment; hTEEPM: Patient group randomized to hTEE with protocolized monitoring; hTEESM: Patient group randomized to hTEE with standard monitoring; ICU: Intensive care unit; LOS: Length of stay; LV: Left ventricle; LVEDA: Left ventricular area at end-diastole; LVESA: Left ventricular area at end-systole; ME asc aortic SAX): Mid-esophageal ascending aortic short-axis view; ME four chamber: Mid-esophageal four chamber view; PDMS: Patient data monitoring system; RV: Right ventricle; RVEDA/LVEDA ratio: Ratio of right ventricular to left ventricular area; SVC Cl: Collapsibility index of superior vena cava; SVC: Superior vena cava; TEE: Transesophageal echocardiography; TG mid SAX: Transgastric midesophageal short-axis view
\end{abstract}

\section{Funding}

This research receives no specific grant from any funding agency in the public, commercial, or not-for-profit sectors. We received 100 detachable hTEE probes for study use free of charge by IMACOR, New York NY, USA. The company is not involved in study design and collection, management, analysis and interpretation of data.

\section{Dissemination}

All trial results whether positive, negative, or neutral will end up in the public domain, preferably in a peer-reviewed publication. The order of authorships for the study main publication will be as follows: TMM, LC, BZ, AB, DB and AL will be the first to seventh, and JT the last author.

\section{Authors' contributions}

LC, SMJ, JT and TMM conceived the study. TMM is the principal investigator, JT acts as study chair and SMJ as study director. BZ, AB and DB were involved in conception and trial design and in drafting of the article. AL provided statistical expertise. All authors were involved in critical revision of the article for important intellectual content and have final approval of the article.

\section{Ethics approval and consent to participate}

This study is carried out in accordance with the protocol and conducted according to international standards of Good Clinical Practice, applicable government regulations and Institutional research policies and procedures. The study has been approved by the Institutional Review Board (IRB) of the Canton of Bern (Kantonale Ethikkommission des Kantons Bern). Any important protocol modifications are reported to the IRB.

Most eligible patients are unable to give consent for the study at the time of ICU admission because of mental incapacity due to the underlying medical condition and treatment (mechanical ventilation). The study is based on assessing parameters during the initial stabilization period immediately after ICU admission; therefore, randomization and study inclusion must occur as soon as possible. Before a patient is enrolled, a physician who is not participating in study must confirm that the interests of the patient are safeguarded and that all inclusion criteria and no exclusion criteria are present. Thereafter, deferred consent involving study inclusion according to the stated criteria in this protocol, followed by the request for a representative's (deferred proxy consent, within $72 \mathrm{~h}$ ) and patient's informed consent (deferred subject consent, as soon as patient's conditions allows) in a later phase, is used. All subjects for this study and their designed representatives receive a consent form describing this study and providing sufficient information for subjects to make an informed decision about their participation in this study. The consent form must be signed by the subject or legally acceptable surrogate, and the investigator-designated research professional obtaining the consent. In cases where the patient's representative's consent to the study and the patient's consent cannot be obtained due to the patient's condition (death, neurological compromise) after provisional study inclusion by proxy consent, the data are used for further analysis. In cases where it is not possible to contact relatives, or if no relatives exist and the patient's consent cannot be obtained due to the patient's condition (death, neurological compromise) after provisional study inclusion by deferred consent, the data are used for further analysis. In the event that a study patient dies before being able to come to an informed decision on study participation and no relatives are available for deferred proxy consent, the collected data are used for analysis [21, 22]. If the patient or relatives deny consent for study inclusion or if the patient or relatives withdraw consent at any time after provisional study inclusion by deferred consent, data are not used for further analysis.

\section{Competing interests}

The authors declare that no support from any organization was received for the submitted work. The Department of Intensive Care Medicine of the University Hospital Bern, Switzerland has, or has had in the past, research contracts with Orion Corporation, Abbott Nutrition International, B. Braun Medical AG, CSEM SA, Edwards Lifesciences Services GmbH, Kenta Biotech Ltd., Maquet Critical Care AB, Omnicare Clinical Research AG and research and development/consulting contracts with Edwards Lifesciences SA, Maquet Critical Care AB, and Nestlé. The Department has received unrestricted educational grants from the following organizations for organizing a quarterly postgraduate educational symposium, the Berner Forum for Intensive Care (until 2015): Fresenius Kabi, gsk, MSD, Lilly, Baxter, astellas, AstraZeneca, B Braun, CSL Behring, Maquet, Novartis, Covidien, Nycomed, Pierre Fabre Pharma AG (formerly known as RobaPharm), Pfizer, Orion Pharma, Bard Medica S.A., Abbott AG, Anandic Medical Systems. The Department has received unrestricted educational grants from the following organizations for organizing bi-annual postgraduate courses in the fields of critical care ultrasound, management of ECMO and mechanical ventilation: Pierre Fabre Pharma AG (formerly known as RobaPharm), Pfizer AG, Bard Medica S.A., Abbott AG, Anandic Medical Systems, PanGas AG Healthcare, Orion Pharma, Bracco, Edwards Lifesciences AG, Hamilton Medical AG, Fresenius Kabi (Schweiz) AG, Getinge Group Maquet AG, Dräger Schweiz AG.

\section{Publisher's Note}

Springer Nature remains neutral with regard to jurisdictional claims in published maps and institutional affiliations.

\section{Author details}

${ }^{1}$ Department of Intensive Care Medicine, University Hospital, and University of Bern, 3010 Bern, Switzerland. ${ }^{2}$ CTU Bern, and Institute of Social and Preventive Medicine (ISPM), University of Bern, Bern, Switzerland.

Received: 5 October 2017 Accepted: 1 June 2018

Published online: 06 July 2018

References

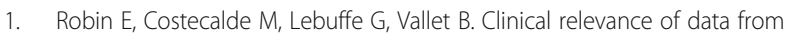
the pulmonary artery catheter. Crit Care. 2006;10(Suppl 3):S3.

2. Ospina-Tascon GA, Cordioli RL, Vincent JL. What type of monitoring has been shown to improve outcomes in acutely ill patients? Intensive Care Med. 2008;34:800-20.

3. Hadian M, Kim HK, Severyn DA, Pinsky MR. Cross-comparison of cardiac output trending accuracy of LiDCO, PiCCO, FloTrac and pulmonary artery catheters. Crit Care. 2010;14:R212.

4. Vincent JL, Rhodes A, Perel A, Martin GS, Della Rocca G, Vallet B, Pinsky MR, Hofer CK, Teboul JL, de Boode WP, et al. Clinical review: update on hemodynamic monitoring-a consensus of 16. Crit Care. 2011;15:229. 
5. Noritomi DT, Vieira ML, Mohovic T, Bastos JF, Cordioli RL, Akamine N, Fischer $\mathrm{CH}$. Echocardiography for hemodynamic evaluation in the intensive care unit. Shock. 2010;34(Suppl 1):59-62.

6. Vignon P, Mentec $H$, Terre S, Gastinne H, Gueret P, Lemaire F. Diagnostic accuracy and therapeutic impact of transthoracic and transesophageal echocardiography in mechanically ventilated patients in the ICU. Chest. 1994;106:1829-34.

7. Jensen MB, Sloth E, Larsen KM, Schmidt MB. Transthoracic echocardiography for cardiopulmonary monitoring in intensive care. Eur J Anaesthesiol. 2004;21:700-7.

8. Vieillard-Baron A, Mayo PH, Vignon P, Cholley B, Slama M, Pinsky MR, McLean A, Choi G, Beaulieu Y, Arntfield R, Koenig S, Colreavy F, Canivet JL, De Backer $\mathrm{D}$. International consensus statement on training standards for advanced critical care echocardiography. Intensive Care Med. 2014;40:654666.

9. Charron C, Vignon P, Prat G, Tonnelier A, Aegerter P, Boles JM, Amiel $\mathrm{JB}$, Vieillard-Baron A. Number of supervised studies required to reach competence in advanced critical care transesophageal echocardiography. Intensive Care Med. 2013;39(6):1019-24.

10. Vignon P, Merz TM, Vieillard-Baron A. Ten reasons for performing hemodynamic monitoring using transesophageal echocardiography. Intensive Care Med. 2017:43(7):1048-1051.

11. Vieillard-Baron A, Slama M, Mayo P, Charron C, Amiel JB, Esterez C, Leleu F, Repesse $X$, Vignon P. A pilot study on safety and clinical utility of a singleuse 72-hour indwelling transesophageal echocardiography probe. Intensive Care Med. 2013:39(4):629-35.

12. Cioccari L, Baur HR, Berger D, Wiegand J, Takala J, Merz TM. Hemodynamic assessment of critically ill patients using a miniaturized transesophageal echocardiography probe. Crit Care. 2013;17:R121.

13. Doig GS, Simpson F. Randomization and allocation concealment: a practical guide for researchers. J Crit Care. 2005;20:187-91. discussion 191-183

14. Vieillard-Baron A, Chergui K, Rabiller A, Peyrouset O, Page B, Beauchet A, Jardin F. Superior vena caval collapsibility as a gauge of volume status in ventilated septic patients. Intensive Care Med. 2004;30:1734-9.

15. Takala J, Dellinger RP, Koskinen K, St Andre A, Read M, Levy M, Jakob SM, Mello PV, Friolet R, Ruokonen E. Development and simultaneous application of multiple care protocols in critical care: a multicenter feasibility study. Intensive Care Med. 2008;34:1401-10.

16. Barthel FM-S, Royston P, Babiker A. A menu-driven facility for complex sample size calculation in randomized controlled trials with a survival or a binary outcome: update. Stata J. 2005;5:123-9.

17. Fine JP, Gray RJ. A proportional hazards model for the subdistribution of a competing risk. J Am Stat Assoc. 1999;94:496-509.

18. Thygesen K, Alpert JS, Jaffe AS, Simoons ML, Chaitman BR, White HD, Katus HA, Lindahl B, Morrow DA, Clemmensen PM, et al. Third universal definition of myocardial infarction. Circulation. 2012;126:2020-35.

19. De Backer D, Biston P, Devriendt J, Madl C, Chochrad D, Aldecoa C, Brasseur A, Defrance P, Gottignies P, Vincent JL. Comparison of dopamine and norepinephrine in the treatment of shock. N Engl J Med. 2010;362:779-89.

20. Malbrain M, De Tavernier B, Haverals S, Slama M, Vieillard-Baron A, Wong A, Poelaert J, Monnet X, Stockman W, Elbers P, Lichtenstein D. Executive summary on the use of ultrasound in the critically ill: consensus report from the 3rd course on acute care ultrasound (CACU). Anaesthesiol Intensive Ther. 2017:49:393-411.

21. Jansen TC, Bakker J, Kompanje EJ. Inability to obtain deferred consent due to early death in emergency research: effect on validity of clinical trial results. Intensive Care Med. 2010;36:1962-5.

22. Jansen TC, Kompanje EJ, Bakker J. Deferred proxy consent in emergency critical care research: ethically valid and practically feasible. Crit Care Med. 2009:37:565-8.

Ready to submit your research? Choose BMC and benefit from:

- fast, convenient online submission

- thorough peer review by experienced researchers in your field

- rapid publication on acceptance

- support for research data, including large and complex data types

- gold Open Access which fosters wider collaboration and increased citations

- maximum visibility for your research: over $100 \mathrm{M}$ website views per year

At BMC, research is always in progress.

Learn more biomedcentral.com/submissions 